\title{
Influence of disorder on transfer characteristics of organic electrochemical transistors
}

Jacob T. Friedlein, Jonathan Rivnay, David H. Dunlap, lain McCulloch, Sean E. Shaheen, Robert R. McLeod, and George G. Malliaras

Citation: Appl. Phys. Lett. 111, 023301 (2017); doi: 10.1063/1.4993776

View online: http://dx.doi.org/10.1063/1.4993776

View Table of Contents: http://aip.scitation.org/toc/apl/111/2

Published by the American Institute of Physics

\section{Articles you may be interested in}

High-performance pentacene OTFT by incorporating Ti in LaON gate dielectric

Applied Physics Letters 111, 023501 (2017); 10.1063/1.4993157

Enhanced sensing of gas molecules by a $99.9 \%$ semiconducting carbon nanotube-based field-effect transistor sensor

Applied Physics Letters 111, 022102 (2017); 10.1063/1.4991970

A flexible graphene terahertz detector

Applied Physics Letters 111, 021102 (2017); 10.1063/1.4993434

Active control and switching of broadband electromagnetically induced transparency in symmetric metadevices Applied Physics Letters 111, 021101 (2017); 10.1063/1.4993428

Development of an ultrafast electron source based on a cold-field emission gun for ultrafast coherent TEM Applied Physics Letters 111, 023101 (2017); 10.1063/1.4991681

Threshold voltage control of electrolyte solution gate field-effect transistor by electrochemical oxidation Applied Physics Letters 111, 013505 (2017); 10.1063/1.4991364

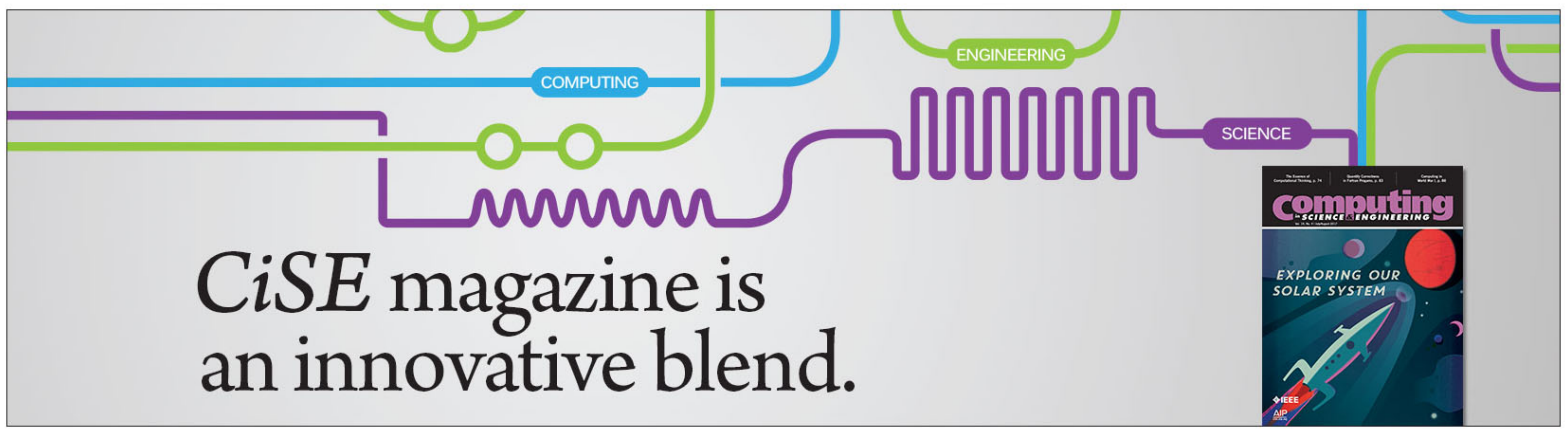




\title{
Influence of disorder on transfer characteristics of organic electrochemical transistors
}

\author{
Jacob T. Friedlein, ${ }^{1}$ Jonathan Rivnay, ${ }^{2}$ David H. Dunlap, ${ }^{3}$ Iain McCulloch, ${ }^{4,5}$ \\ Sean E. Shaheen, ${ }^{1,6}$ Robert R. McLeod, ${ }^{1}$ and George G. Malliaras ${ }^{7}$ \\ ${ }^{1}$ Department of Electrical, Computer, and Energy Engineering, University of Colorado, 425 UCB, \\ Boulder, Colorado 80309, USA \\ ${ }^{2}$ Department of Biomedical Engineering, Northwestern University, 2145 Sheridan Road, Evanston, \\ Illinois 60208-3109, USA \\ ${ }^{3}$ Department of Physics and Astronomy, University of New Mexico, 1919 Lomas Blvd. NE, Albuquerque, \\ New Mexico 87131, USA \\ ${ }^{4}$ Department of Chemistry and Centre for Plastic Electronics, Imperial College London, London SW7 $2 A Z$, \\ United Kingdom \\ ${ }^{5}$ King Abdullah University of Science and Technology, SPERC, Thuwal 23955-6900, Saudi Arabia \\ ${ }^{6}$ Department of Physics, University of Colorado, 390 UCB, Boulder, Colorado 80309, USA \\ ${ }^{7}$ Department of Bioelectronics, Ecole Nationale Supérieure des Mines CMP-EMSE MOC Gardanne 13541, \\ France
}

(Received 16 May 2017; accepted 26 June 2017; published online 13 July 2017)

\begin{abstract}
Organic electrochemical transistors (OECTs) are receiving a great deal of attention as transducers of biological signals due to their high transconductance. A ubiquitous property of these devices is the non-monotonic dependence of transconductance on gate voltage. However, this behavior is not described by existing models. Using OECTs made of materials with different chemical and electrical properties, we show that this behavior arises from the influence of disorder on the electronic transport properties of the organic semiconductor and occurs even in the absence of contact resistance. These results imply that the non-monotonic transconductance is an intrinsic property of OECTs and cannot be eliminated by device design or contact engineering. Finally, we present a model based on the physics of electronic conduction in disordered materials. This model fits experimental transconductance curves and describes strategies for rational material design to improve OECT performance in sensing applications. Published by AIP Publishing. [http://dx.doi.org/10.1063/1.4993776]
\end{abstract}

Organic electrochemical transistors (OECTs) use ions injected from an electrolyte to change the doping state, and hence the electrical conductivity, of an organic semiconductor channel. ${ }^{1}$ These ions are injected into the channel by a voltage at a gate electrode immersed in the electrolyte. Source and drain electrodes establish electrical contact to the semiconducting film and measure the drain current that flows in the channel. OECTs can be designed for either accumulation mode or depletion mode operation. In accumulation mode OECTs, applying a gate voltage turns the OECT on by injecting ionic dopants into the channel, thus increasing the electronic carrier concentration. ${ }^{2-5}$ In depletion mode devices, applying a gate voltage turns the OECT off by injecting ions that compensate native dopants and reduce the carrier concentration. ${ }^{1,6,7}$

OECTs can transduce voltage signals in the gate circuit to changes in the drain current with significant amplification of signal power. ${ }^{8,9}$ This makes OECTs useful in many biosensing applications, ${ }^{10}$ especially the recording of sub-mV electrophysiological signals. ${ }^{11-13}$ Transistor signal transduction is characterized by transfer curves, which describe the dependence of drain current on gate voltage. The derivative of the transfer curve is transconductance, $g_{m}$, which is a figure-of-merit for describing transduction efficiency. The volumetric doping in OECTs endows these devices with very high transconductance values. ${ }^{9,14}$ However, OECT transconductance has a non-monotonic dependence on gate voltage, decreasing at both high and low gate voltages. This behavior has been observed since the first OECTs were reported in the seminal work of Wrighton et al. in $1984 .{ }^{15}$ It is a ubiquitous property of OECTs, reported in devices made with different organic semiconductors-including polythiophenes, polyaniline, polypyrrole, and polyacetylene $\mathrm{e}^{16,17}$ as well as different source-drain electrodes-such as gold, ${ }^{9}$ poly(3,4-ethylenedioxythiophene):poly(styrenesulfonate) (PEDOT:PSS), ${ }^{18}$ and carbon. ${ }^{19}$ Moreover, non-monotonic transconductance occurs for various electrode geometries ${ }^{15,18,20,21}$ and a wide range of fabrication technologies-including orthogonal photolithography, ${ }^{22}$ parylene-based photolithography, ${ }^{9}$ screen printing, ${ }^{23}$ and inkjet printing. ${ }^{24}$

Despite numerous reports of bell-shaped transconductance in OECTs, existing models do not predict this behavior. For example, the model of Bernards predicts that transconductance is constant in the linear regime and decreases linearly with gate voltage in the saturation regime. ${ }^{1}$ Other models for OECTs assume a non-linear relationship between conductivity and charge carrier concentration in the polymer, ${ }^{7,25}$ and Friedlein et al. noted that hopping transport would cause such a relationship. ${ }^{25}$ Although these models fit experimental results showing non-constant transconductance in the linear regime, they do not predict a bell-shaped dependence of transconductance on gate voltage. Nonetheless, the partial success of these models suggests that material disorder causes non-linear transfer curves in OECTs.

Another possible explanation for this behavior is a gate-dependent contact resistance. Gate-dependent contact 
resistance causes bell-shaped transconductance in organic FETs ${ }^{26,27}$ and this effect was recently explored in OECTs. ${ }^{28}$ Kaphle et al. used an empirical model for the dependence of contact resistance on gate voltage and showed that this can lead to a non-monotonic transconductance. These results imply that this behavior is not an intrinsic property of OECTs, but rather a result of poor electrical contact to the channel.

To examine the cause of the non-monotonic transconductance in OECTs, we fabricated OECTs on glass substrates with gold source and drain contacts, and we included probes for voltage measurements at five positions along the OECT channel, as shown in Fig. 1(a). We used these probes for two purposes. First, we measured the voltage along the OECT channel and extracted the voltage drop at the source electrode due to contact resistance. Second, we made 4-wire conductance measurements by using the outermost voltage probes for the sense contacts while using the source and drain as force contacts in a standard Kelvin configuration. The OECT channels consisted of either PEDOT:PSS or p(g2T-TT) as the semiconductor [see Fig. 1(b)]. PEDOT:PSS is a two-phase polymer salt where the semiconducting PEDOT phase is doped by PSS, whereas $\mathrm{p}$ (g2T-TT) is a single-phase polymer without any native dopants. ${ }^{4}$ This material distinction allows fabrication of both depletion and accumulation mode OECTs and hence provides an opportunity to probe the generality of our conclusions. We used a typical OECT geometry with $100 \mathrm{mM} \mathrm{NaCl}$ in water as the electrolyte and a $12.5 \mathrm{~mm}^{2} \mathrm{Ag} /$ $\mathrm{AgCl}$ pellet as the gate electrode. Additional experimental details are available in the supplementary material.

Figure 2(a) shows the contact resistance for a PEDOT:PSS-based transistor. Although it is not possible to measure contact resistance at the drain electrode because of depletion effects, if we assume that it is nearly the same as that at the source electrode, we find that the total contact resistance in the PEDOT:PSS-based OECT could be up to
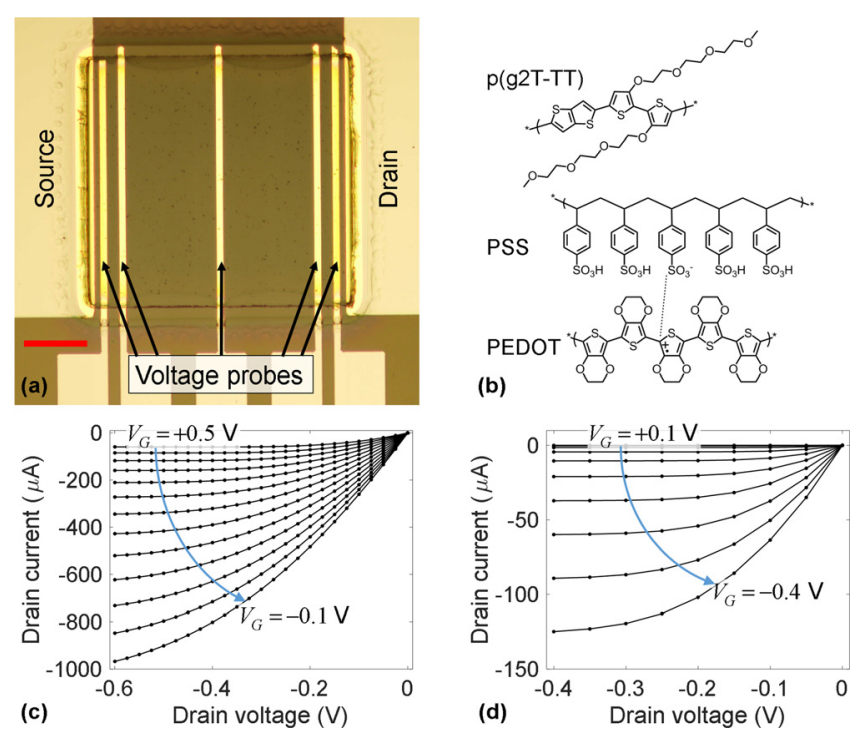

FIG. 1. OECT geometry, polymer structures, and steady-state characteristics. (a) Bright-field image of an OECT with voltage probes. The scale bar is $50 \mu \mathrm{m}$. (b) Structure of poly(2-(3,3-bis(2-(2-(2-methoxyethoxy)ethoxy) ethoxy)-[2,2'-bithiophen]-5-yl)thieno[3,2-b]thiophene)—abbreviated p(g2TTT) (top) and PEDOT:PSS (bottom). (c) and (d) Output curves of an OECT with (c) a PEDOT:PSS channel and (d) a p(g2T-TT) channel.
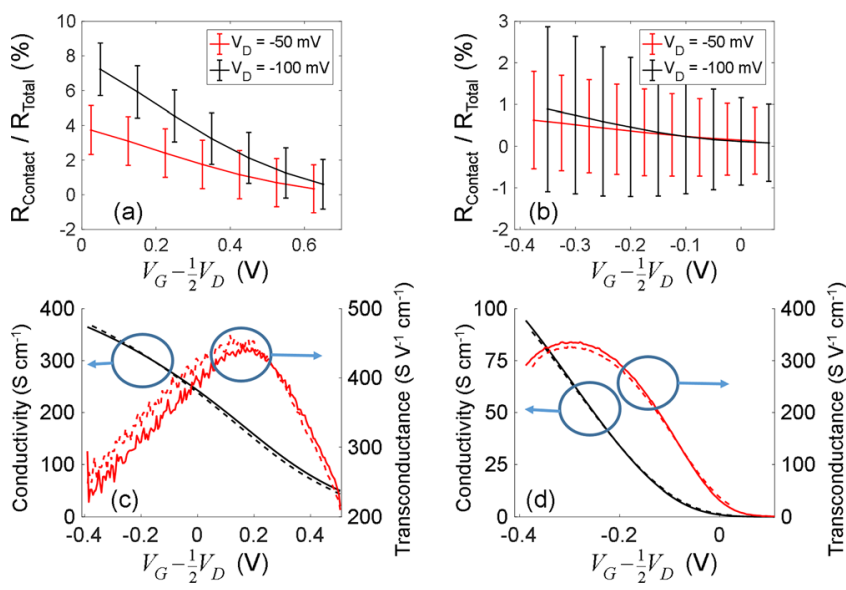

FIG. 2. Effect of contact resistance. (a) and (b) Contact resistance at the source electrode divided by the total resistance (contact resistance plus channel resistance) for (a) a PEDOT:PSS-based OECT and (b) a $\mathrm{p}(\mathrm{g} 2 \mathrm{~T}-$ TT)-based OECT. (c) and (d) Channel conductivity and normalized transconductance $\left[g_{m}^{\prime} \equiv g_{m} /\left|V_{D}\right| \times L /(W h)\right.$, where $V_{D}$ is the drain voltage and $L, W$, and $h$ are the channel length, width, and thickness, respectively] for (c) a PEDOT:PSS-based OECT and (d) a p(g2T-TT)-based OECT. In (c) and (d), dashed lines are 4-wire measurements, and solid lines are 2-wire measurements. Error bars in (a) and (b) are calculated as described in the supplementary material. In (c), both the 2-wire and 4-wire measurements were made with $V_{D}=-10 \mathrm{mV}$. In (d), $V_{D}=-25 \mathrm{mV}$ for the 2-wire measurements and $\mathrm{V}_{\mathrm{D}}=-50 \mathrm{mV}$ for the 4-wire measurements. In (a)-(d), instead of using the gate voltage, $V_{G}$, we have used the median gatechannel voltage, $V_{G}-(1 / 2) V_{D}$, on the horizontal axis to facilitate comparison between measurements made at different drain voltages. Both devices have $W=200 \mu \mathrm{m} \times L=200 \mu \mathrm{m}$ for 2-wire measurements and $L=180 \mu \mathrm{m}$ for 4-wire measurements. For PEDOT:PSS, $h \approx 100 \mathrm{~nm}$, and for $\mathrm{p}(\mathrm{g} 2 \mathrm{~T}-$ TT), $h \approx 85 \mathrm{~nm}$.

$20 \%$ of the total resistance (see Fig. S4 in the supplementary material). Therefore, in PEDOT:PSS-based OECTs with this $\mathrm{W} / \mathrm{L}$ ratio geometry, contact resistance could influence the transfer characteristics. In contrast to the PEDOT:PSS-based OECT, the p(g2T-TT)-based OECT [Fig. 2(b)] has contact resistance which contributes negligibly to the total resistance. Figures 2(c) and 2(d) show how contact resistance affects the transfer and transconductance curves. Contact resistance has an effect on the transfer curves for 2-wire measurements, but it has no effect on 4-wire measurements because separate electrodes are used for supplying voltage and measuring current, as in typical four point probe measurements. By comparing the 2-wire and 4-wire measurements, one can see that contact resistance in PEDOT:PSS-based OECTs reduces the transconductance by about $10 \%$ at gate voltages less than $0.2 \mathrm{~V}$. Nonetheless, non-monotonic transconductance is evident in the 4-wire measurements for both PEDOT:PSS and $\mathrm{p}(\mathrm{g} 2 \mathrm{~T}-\mathrm{TT})$, even though these measurements are unaffected by contact resistance. Therefore, contact resistance is not the primary cause of non-monotonic transconductance. Transmission line measurements support this conclusion by showing that contact resistance contributes negligibly to the total resistance in long-channel devices. For instance, in a device with a channel width of $10 \mu \mathrm{m}$ and a channel length of $250 \mu \mathrm{m}$, the extracted contact resistance is less than $2 \%$ of the total resistance, but the device still has a markedly nonmonotonic transconductance (Fig. S5 in the supplementary material).

Because we found that contact resistance is not the primary cause of the non-ideal transfer curves, we modeled 
OECT behavior using a modified form of Ambegaokar, Halperin, and Langer's theory for hopping conduction in disordered materials. ${ }^{29}$ Figure 3 shows the fit between this model and the experimental data. Briefly, the model assumes a Gaussian-shaped energy density of states (DOS) and a Miller-Abrahams form of detailed balance for transition rates between nearest-neighbor hopping sites. ${ }^{30}$ The present model is discussed in more detail in the supplementary material and predicts that as the gate voltage is made more negative, more holes are added to the semiconductor, filling the DOS. At first, when the DOS is much less than half full, adding holes increases both the hole concentration and the hole mobility. In this regime, transconductance increases as the gate voltage is made more negative. As the DOS becomes nearly half full, the rate of increase of both hole concentration and mobility slows, causing a decrease in transconductance as the gate voltage is made more negative. When the DOS is more than half full, adding more holes decreases hole mobility, and the transconductance becomes negative (see Fig. S1 in the supplementary material). These predictions qualitatively agree with other theoretical and experimental studies that show a non-linear relationship between conductivity and charge carrier concentration. ${ }^{31-35}$

The proposed model fits the transconductance curves of both PEDOT:PSS-based and p(g2T-TT)-based OECTs with four free parameters. As predicted by the model, these polymers exhibit an initial increase and subsequent decrease of transconductance as gate voltage becomes more negative. The model further predicts that at more negative gate voltages, the transconductance will become negative, but we were not able to observe this behavior because we had to avoid the over-oxidation of PEDOT and the electrolysis of water. Other polymer/electrolyte systems are stable at more negative gate voltages, and OECTs with those materials do exhibit the full range of predicted features-including negative transconductance. ${ }^{16,36}$ Extracted values for the four fit parameters are listed in Table I; $\sigma_{0}$ is the polymer conductivity at $V_{G}=0 \mathrm{~V}$ for PEDOT:PSS and at $V_{G}=-0.4 \mathrm{~V}$ for $\mathrm{p}(\mathrm{g} 2 \mathrm{~T}-\mathrm{TT}) ; E_{0}$ is the energetic position of the peak of the DOS with respect to the $\mathrm{Ag} / \mathrm{AgCl}$ electrode; $\sigma_{D O S}{ }^{2}$ is the DOS variance; and $\eta$ is the critical probability of bond connectivity required for percolation.

The extracted $\sigma_{0}$ values are similar to the reported values for PEDOT:PSS and p(g2T-TT) ${ }^{4,14,28}$ and the extracted DOS widths $\left(\sigma_{D O S}\right)$ are about one order of magnitude larger
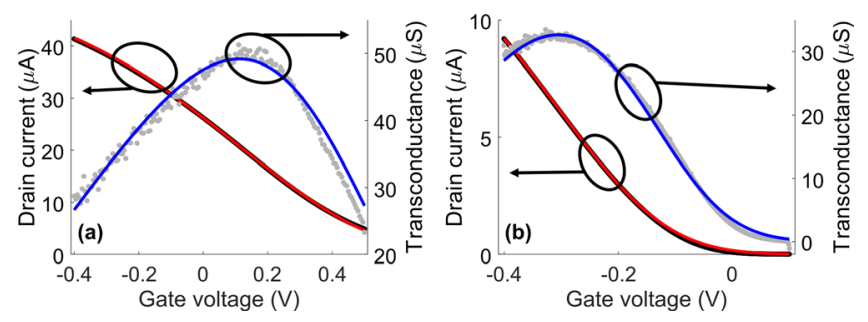

FIG. 3. Disorder model fit to OECT transconductance for (a) a PEDOT:PSS-based OECT and (b) a p(g2T-TT)-based OECT. In both figures, the solid blue curve is the fit to the transconductance, the solid red curve is the model prediction for the drain current, the black dots are the experimentally measured drain current, and the gray dots are the experimentally measured transconductance. $V_{D}=-10 \mathrm{mV}$ and 4-wire sensing is used for both (a) and (b).
TABLE I. Fit parameters for the disorder model.

\begin{tabular}{lcccc}
\hline \hline & $\sigma_{0}(\mathrm{~S} / \mathrm{cm})$ & $\sigma_{D O S}(\mathrm{meV})$ & $E_{0}(\mathrm{meV})$ & $\eta(\%)$ \\
\hline PEDOT:PSS & $278 \pm 0.6$ & $950 \pm 30$ & $-830 \pm 60$ & $0.1 \pm 0.01$ \\
$\mathrm{p}(\mathrm{g} 2 \mathrm{~T}-\mathrm{TT})$ & $96 \pm 0.5$ & $410 \pm 10$ & $-640 \pm 10$ & $1.0 \pm 0.1$ \\
\hline \hline
\end{tabular}

than that reported for dry films of polythiophenes. ${ }^{37,38}$ However, DOS widths for electrochemically doped polythiophenes can broaden significantly. ${ }^{36,39,40}$ The critical bond connectivity, $\eta$, extracted by the proposed model for $\mathrm{p}(\mathrm{g} 2 \mathrm{~T}$ TT) is about one order of magnitude smaller than expected for close-packed spheres, and for PEDOT:PSS, it is about two orders of magnitude smaller than expected. ${ }^{29}$ However, $\eta$ decreases strongly as the aspect ratio of conducting structures increases, ${ }^{41}$ and conduction in polymers depends on high aspect ratio structures due to carrier delocalization along polymer backbones ${ }^{42}$ and anisotropic phase segregation. ${ }^{43}$ Therefore, $\eta$ is expected to be much smaller in polymers than in systems composed of close-packed spheres.

Non-monotonic transconductance induced by material disorder has several implications for OECT research. For instance, if contact resistance were the primary cause of the non-monotonic transconductance, the decrease in transconductance with more negative gate voltages could be prevented by changing the metals used for source/drain contacts and engineering the interfaces at these contacts. However, because non-monotonic transconductance is an intrinsic property of OECTs, such strategies will not eliminate the transconductance decrease at negative gate voltages. Our results suggest that researchers developing new materials should focus not only on maximizing transconductance but also on ensuring that high transconductance occurs over a broad voltage window. In this respect, our model predicts a tradeoff in OECT performance; decreasing the DOS width not only increases the peak transconductance, conductance, and mobility but also decreases the width of the voltage range over which transconductance remains near its peak. However, our model suggests that one can work around this tradeoff by engineering $\eta$ because as $\eta$ decreases, the transconductance peak becomes higher without becoming narrower (Fig. S1 in the supplementary material). Therefore, designing materials with highly anisotropic conducting structures could improve OECT performance. Unfortunately, it is not clear in the present model whether anisotropy is most important at the molecular level of carrier delocalization or at the microscopic level of phase segregation. Finally, new materials should be designed such that the maximum transconductance occurs at voltages within the stable operating regime of the transistor/ electrolyte system. This could be accomplished by adjusting the electronegativity of side-chain constituents to shift the HOMO level of the semiconducting polymer. ${ }^{44}$ Altogether, the present model suggests several design strategies for OECT materials: (1) increasing the anisotropy of conducting structures, (2) adjusting the HOMO level so that peak transconductance occurs within the operational voltage range, and (3) tailoring the DOS width on a case-by-case basis according to the tradeoff between maximizing peak transconductance and broadening the window of high transconductance. 
In conclusion, we fabricated accumulation-mode and depletion-mode OECTs based on two different polymer semiconductors, and we showed that both materials exhibit non-monotonic transconductance. Using 4-wire conductance measurements and transmission line measurements, we showed that non-monotonic transconductance occurs even in the absence of contact resistance. We demonstrated that a model based on hopping transport in disordered materials can explain the transconductance of OECTs, thus supporting the conclusion that non-monotonic transconductance is an intrinsic property in OECTs. The model presented here provides predictions about how material parameters, such as delocalization length, HOMO level, and DOS width, affect OECT transfer characteristics. These results will inform the design of materials for improved OECT performance in sensor applications.

See supplementary material for details about device fabrication, contact resistance measurements, transmission line measurements, and model used to describe transport in disordered materials.

The authors of this letter would like to thank Rene Lujan (PARC) for fabrication of transistors with voltage probes and Marcel Brändlein for the fabrication and characterization of the transistors used for the transmission line method measurements.

This letter is based upon work supported by the National Science Foundation Graduate Research Fellowship under Grant No. DGE 1144083 and NSF Grant No. NSF EPMD 1509909.

J.T.F. acknowledges support from the Graduate Assistantships in Areas of National Need Award No. P200A120063 and the NSF GRFP Award No. DGE 1144083. S.E.S. acknowledges support from the National Science Foundation Grant No. DMR-1006930. R.R.M. acknowledges support from the National Science Foundation Grant CAREER (No. ECCS 0847390). R.R.M. and S.E.S. acknowledge support from the National Science Foundation Grant No. ECCS 1509909.

${ }^{1}$ D. A. Bernards and G. G. Malliaras, Adv. Funct. Mater. 17, 3538 (2007).

${ }^{2}$ S. Inal, J. Rivnay, P. Leleux, M. Ferro, M. Ramuz, J. C. Brendel, M. M. Schmidt, M. Thelakkat, and G. G. Malliaras, Adv. Mater. 26, 7450 (2014).

${ }^{3}$ E. Zeglio, J. Eriksson, R. Gabrielsson, N. Solin, and O. Inganäs, Adv. Mater. 29, 1605787 (2017).

${ }^{4}$ A. Giovannitti, D.-T. Sbircea, S. Inal, C. B. Nielsen, E. Bandiello, D. A. Hanifi, M. Sessolo, G. G. Malliaras, I. McCulloch, and J. Rivnay, Proc. Natl. Acad. Sci. 113, 12017 (2016).

${ }^{5}$ A. Giovannitti, C. B. Nielsen, D.-T. Sbircea, S. Inal, M. Donahue, M. R. Niazi, D. A. Hanifi, A. Amassian, G. G. Malliaras, J. Rivnay, and I. Mcculloch, Nat. Commun. 7, 13066 (2016).

${ }^{6}$ B. D. Nilsson, M. Chen, T. Kugler, T. Remonen, M. Armgarth, and M. Berggren, Adv. Mater. 14, 51 (2002).

${ }^{7}$ N. D. Robinson, P.-O. Svensson, D. Nilsson, and M. Berggren, J. Electrochem. Soc. 153, H39 (2006).

${ }^{8}$ J. Rivnay, P. Leleux, M. Sessolo, D. Khodagholy, T. Herve, M. Fiocchi, and G. G. Malliaras, Adv. Mater. 25, 7010 (2013).

${ }^{9}$ D. Khodagholy, J. Rivnay, M. Sessolo, M. Gurfinkel, P. Leleux, L. H. Jimison, E. Stavrinidou, T. Herve, S. Sanaur, R. M. Owens, and G. G. Malliaras, Nat. Commun. 4, 2133 (2013).
${ }^{10}$ X. Strakosas, M. Bongo, and R. M. Owens, J. Appl. Polym. Sci. 132, 41735 (2015).

${ }^{11}$ P. Leleux, J. Rivnay, T. Lonjaret, J.-M. Badier, C. Bénar, T. Hervé, P. Chauvel, and G. G. Malliaras, Adv. Healthcare Mater. 4, 142 (2015).

${ }^{12}$ D. Khodagholy, T. Doublet, P. Quilichini, M. Gurfinkel, P. Leleux, A. Ghestem, E. Ismailova, T. Hervé, S. Sanaur, C. Bernard, and G. G. Malliaras, Nat. Commun. 4, 1575 (2013).

${ }^{13}$ A. Campana, T. Cramer, D. T. Simon, M. Berggren, and F. Biscarini, Adv. Mater. 26, 3874 (2014).

${ }^{14}$ J. Rivnay, P. Leleux, M. Ferro, M. Sessolo, A. Williamson, D. A. Koutsouras, D. Khodagholy, M. Ramuz, X. Strakosas, R. M. Owens, C. Benar, J.-M. Badier, C. Bernard, and G. G. Malliaras, Sci. Adv. 1, e1400251 (2015).

${ }^{15}$ H. S. White, G. P. Kittlesen, and M. S. Wrighton, J. Am. Chem. Soc. 106, 5375 (1984).

${ }^{16}$ D. Ofer, R. M. Crooks, and M. S. Wrighton, J. Am. Chem. Soc. 112, 7869 (1990).

${ }^{17}$ D. Ofer, L. Y. Park, R. R. Schrock, and M. S. Wrighton, Chem. Mater. 3, 573 (1991).

${ }^{18}$ E. Bihar, Y. Deng, T. Miyake, M. Saadaoui, G. G. Malliaras, and M. Rolandi, Sci. Rep. 6, 27582 (2016).

${ }^{19}$ T. Blaudeck, P. A. Ersman, M. Sandberg, S. Heinz, A. Laiho, J. Liu, I. Engquist, M. Berggren, and R. R. Baumann, Adv. Funct. Mater. 22, 2939 (2012).

${ }^{20}$ S. Chao and M. S. Wrighton, J. Am. Chem. Soc. 109, 2197 (1987).

${ }^{21}$ P. Andersson Ersman, D. Nilsson, J. Kawahara, G. Gustafsson, and M. Berggren, Org. Electron. 14, 1276 (2013).

${ }^{22}$ S. Zhang, E. Hubis, C. Girard, P. Kumar, J. DeFranco, and F. Cicoira, J. Mater. Chem. C 4, 1382 (2016).

${ }^{23}$ G. Scheiblin, R. Coppard, R. M. Owens, P. Mailley, and G. G. Malliaras, Adv. Mater. Technol. 2, 1600141 (2017).

${ }^{24}$ M. Demelas, E. Scavetta, L. Basiricò, R. Rogani, and A. Bonfiglio, Appl. Phys. Lett. 102, 193301 (2013).

${ }^{25}$ J. T. Friedlein, S. E. Shaheen, G. G. Malliaras, and R. R. McLeod, Adv. Electron. Mater. 1, 1500189 (2015).

${ }^{26}$ E. G. Bittle, J. I. Basham, T. N. Jackson, O. D. Jurchescu, and D. J. Gundlach, Nat. Commun. 7, 10908 (2016).

${ }^{27}$ T. J. Richards and H. Sirringhaus, J. Appl. Phys. 102, 94510 (2007).

${ }^{28}$ V. Kaphle, S. Liu, A. Al-Shadeedi, C.-M. Keum, and B. Lüssem, Adv. Mater. 28, 8766 (2016).

${ }^{29}$ V. Ambegaokar, B. I. Halperin, and J. S. Langer, Phys. Rev. B 4, 2612 (1971).

${ }^{30}$ N. Tessler, Y. Preezant, N. Rappaport, and Y. Roichman, Adv. Mater. 21, 2741 (2009).

${ }^{31}$ S. Ihnatsenka, X. Crispin, and I. V. Zozoulenko, Phys. Rev. B 92, 35201 (2015).

${ }^{32}$ V. I. Arkhipov, E. V. Emelianova, P. Heremans, and H. Bässler, Phys. Rev. B 72, 235202 (2005)

${ }^{33}$ J. Zhou, Y. C. Zhou, X. D. Gao, C. Q. Wu, X. M. Ding, and X. Y. Hou, J. Phys. D: Appl. Phys. 42, 35103 (2009).

${ }^{34}$ R. Coehoorn, W. F. Pasveer, P. A. Bobbert, and M. A. J. Michels, Phys. Rev. B: Condens. Matter Mater. Phys. 72, 155206 (2005).

${ }^{35}$ J. Mort, S. Grammatica, D. J. Sandman, and A. Troup, J. Electron. Mater. 9, 411 (1980).

${ }^{36}$ B. D. Paulsen and C. D. Frisbie, J. Phys. Chem. C 116, 3132 (2012).

${ }^{37}$ P. Pingel, R. Schwarzl, and D. Neher, Appl. Phys. Lett. 100, 143303 (2012).

${ }^{38}$ G. Garcia-Belmonte, P. P. Boix, J. Bisquert, M. Sessolo, and H. J. Bolink, Sol. Energy Mater. Sol. Cells 94, 366 (2010).

${ }^{39}$ G. Garcia-Belmonte, E. V. Vakarin, J. Bisquert, and J. P. Badiali, Electrochim. Acta 55, 6123 (2010).

${ }^{40}$ V. I. Arkhipov, P. Heremans, E. V. Emelianova, and H. Bässler, Phys. Rev. B 71, 45214 (2005).

${ }^{41}$ M. A. Klatt, G. E. Schröder-Turk, and K. Mecke, J. Stat. Mech. Theory Exp. 2017, 023302.

${ }^{42}$ S. Stafström and J. L. Brédas, Phys. Rev. B 38, 4180 (1988).

${ }^{43}$ A. M. Nardes, M. Kemerink, R. A. J. Janssen, J. A. M. Bastiaansen, N. M. M. Kiggen, B. M. W. Langeveld, A. J. J. M. van Breemen, and M. M. de Kok, Adv. Mater. 19, 1196 (2007).

${ }^{44}$ J. Hou, H.-Y. Chen, S. Zhang, and Y. Yang, J. Phys. Chem. C 113, 21202 (2009). 